\title{
Coexpression of vesicular glutamate transporters 1 and 2, glutamic acid decarboxylase and calretinin in rat entorhinal cortex
}

\author{
Floris G. Wouterlood • Cathrin B. Canto · Verena Aliane · Amber J. Boekel • \\ Jens Grosche · Wolfgang Härtig · Jeroen A. M. Beliën · Menno P. Witter
}

Received: 21 June 2007/Accepted: 8 October 2007/Published online: 27 October 2007

(C) Springer-Verlag 2007

\begin{abstract}
We studied the distribution and coexpression of vesicular glutamate transporters (VGluT1, VGluT2), glutamic acid decarboxylase (GAD) and calretinin (CR, calcium-binding protein) in rat entorhinal cortex, using immunofluorescence staining and multichannel confocal laser scanning microscopy. Images were computer processed and subjected to automated 3D object recognition, colocalization analysis and 3D reconstruction. Since the VGluTs (in contrast to CR and GAD) occurred in fibers and axon terminals only, we focused our attention on these neuronal processes. An intense, punctate VGluT1-staining occurred everywhere in the entorhinal cortex. Our computer program resolved these punctae as small 3D objects. Also VGluT2 showed a punctate immunostaining pattern, yet with half the number of 3D objects per tissue volume compared with VGluT1, and with statistically significantly larger 3D objects. Both VGluTs were distributed homogeneously across cortical layers, with in MEA VGluT1 slightly more densely
\end{abstract}

F. G. Wouterlood $(\bowtie) \cdot$ C. B. Canto · M. P. Witter Graduate School of Neurosciences, Research Institute Neuroscience, Department of Anatomy \& Neurosciences, Vrije Universiteit Medical Center, Rm MF-G-136, P.O. Box 7057, 1007 MB Amsterdam, The Netherlands e-mail: FG.Wouterlood@vumc.nl

\section{Aliane}

Graduate School of Neurosciences, Research Institute Neuroscience, Department of Anatomy \& Neurosciences, Vrije Universiteit Medical Center,

P.O. Box 7057, 1007 MB Amsterdam, The Netherlands

\section{A. J. Boekel}

Graduate School of Neurosciences, Research Institute

Neuroscience, Department of Anatomy \& Neurosciences, Vrije Universiteit Medical Center, Rm MF-H-132,

P.O. Box 7057, 1007 MB Amsterdam, The Netherlands distributed than in LEA. The distribution pattern and the size distribution of GAD 3D objects resembled that of VGluT2. CR-immunopositive fibers were abundant in all cortical layers. In double-stained sections we noted ample colocalization of CR and VGluT2, whereas coexpression of CR and VGluT1 was nearly absent. Also in triple-staining experiments (VGluT2, GAD and CR combined) we noted coexpression of VGluT2 and CR and, in addition, frequent coexpression of GAD and CR. Modest colocalization occurred of VGluT2 and GAD, and incidental colocalization of all three markers. We conclude that the CR-containing axon terminals in the entorhinal cortex belong to at least two subpopulations of CRneurons: a glutamatergic excitatory and a GABAergic inhibitory.

Keywords Parahippocampal region .

Hippocampal region - Axon terminal markers .

Confocal laser scanning · Neuroanatomical tracing ·

Excitation and inhibition

J. Grosche · W. Härtig

Departments of Neurophysiology,

University of Leipzig, Jahnallee 59,

4109 Leipzig, Germany

W. Härtig

Neurochemistry, Paul Flechsig Institute for Brain Research,

University of Leipzig, Jahnallee 59, 4109 Leipzig, Germany

J. Grosche

Interdisciplinary Center of Clinical Research (IZKF),

Faculty of Medicine, University of Leipzig, Leipzig, Germany

J. A. M. Beliën

Department of Pathology, VU University Medical Center,

Amsterdam, The Netherlands

C. B. Canto - M. P. Witter

Centre for the Biology of Memory, NTNU, Trondheim, Norway 


\section{Introduction}

The entorhinal cortex (EC) takes a major position in the medial temporal lobe as an interface between association cortices, peri- and postrhinal cortices and the hippocampus (Burwell and Witter 2002; Witter and Amaral 2004). The multimodal sensory information received by the EC is processed and via output neurons in the superficial layers projected to the hippocampus (Steward and Scoville 1976; Gloveli et al. 1997). Hippocampal projections to the deep layers of EC contribute return connectivity (Kloosterman et al. 2004). The entorhino-hippocampal circuitry is completed by intrinsic neurons connecting deep to superficial layers of EC (Gloveli et al. 2001; van Haeften et al. 2003). The entorhinal cortex is also reciprocally connected with widespread cortical multimodal association areas, either directly or mediated by way of the perirhinal and postrhinal cortices (Insausti et al. 1997; Swanson and Köhler 1986; Burwell 2000). The cycling and processing of information through these circuits, also known as reverberation, has been proposed essential for the storage of information in the multimodal sensory areas for later retrieval, i.e. memory (Buzsáki 1996).

At various levels in this network, interneurons are at work resulting in the synchronization and inhibition of the activity of the projection neurons (Jones and Buhl 1993; Gloveli et al. 1997; Alonso 2002; Kumar and Buckmaster 2006). Already Lorente de Nó (1933) noted in Golgi-silver stained material a large variety of nonpyramidal neurons in the EC and he depicted several of them with impressive local axon collateral distributions. What are the activity footprint and the chemical identity of all these interneurons? Intracellular neurophysiological studies by diverse groups have identified various physiological types (see review in Alonso 2002). GABAergic and peptidergic populations of interneurons have been identified (see review in Wouterlood 2002). Entorhinal interneurons express, amongst others, calcium-binding proteins such as parvalbumin (PV), calretinin (CR) and calbindin.

In spite of all immunohistochemical studies the chemical identity of various interneurons remains enigmatic. In contrast to entorhinal PV neurons that are without exception GABAergic (Wouterlood et al. 1995), many entorhinal CRimmunopositive neurons do not coexpress GABA (Miettinen et al. 1996, 1997; Wouterlood et al. 2000). Many CR-immunopositive axon terminals in EC even show in the electron microscope features common to excitatory synaptic contacts (Wouterlood et al. 2000). These observations triggered us to propose that a subpopulation of the CR neurons in EC might be excitatory instead of inhibitory. In the present study, we investigated the possible glutamatergic phenotype of these CR-positive, GABA-negative cells. Antibodies against the vesicular glutamate transporters 1 and 2 were selected for this purpose since antibodies against glutamate and its metabolic enzyme glutaminase unfortunately recognize also metabolic glutamate in non-glutamatergic neurons (Ottersen and Storm-Mathisen 1984; Fujiyama et al. 2001). Vesicular glutamate transporters have been established as specific markers for nerve terminals conducting glutamatergic neurotransmission (Ni et al. 1994; Bellocchio et al. 1998, 2000; Aihara et al. 2000; Takamori et al. 2001; Fremeau et al. 2001). We studied the distribution of these markers in the lateral and medial subdivisions of the EC, and we looked for coexpression with CR. Since we are dealing with the possibility of GABAergic and non-GABAergic subpopulations of CR cells (Wouterlood et al. 2000) we also examined whether GABA is present in CR- or VGluT immunoreactive boutons. For this purpose we applied an antibody against glutamic acid decaboxylase (GAD; Oertel et al. 1981). Considerations like resolution, efficiency, confidence and speed of analysis led us to select multilabel confocal laser scanning followed by deconvolution, 3D reconstruction and automated $3 \mathrm{D}$ particle recognition as the method of choice (Wouterlood et al. 2007a). Part of the present material was published in abstract form as a preliminary report (Wouterlood et al. 2003).

\section{Materials and methods}

\section{Experimental animals}

Brain sections were used from 11 female Wistar rats (weight 200-220 g: Harlan Centraal Proefdierbedrijf Zeist, The Netherlands), supplemented with sections belonging to 13 rat brains used in previous immunostaining projects. Animals were handled according to the rules and directives set by the Dutch Government and by University and European Community regulations on animal well-being.

All animals were sacrificed by intraperitoneal injection with an overdose of sodium pentobarbital (Nembutal, i.p. $60 \mathrm{mg} / \mathrm{kg}$ body weight; Sanofi Sante, Maassluis, The Netherlands). They were rapidly perfused transcardially with $100 \mathrm{ml}$ of a $0.9 \%$ solution of $\mathrm{NaCl}$, followed by $500 \mathrm{ml}$ of a mixture of freshly depolymerized $4 \%$ paraformaldehyde and $0.1 \%$ glutaraldehyde (Merck, Darmstadt, Germany) in $100 \mathrm{mM}$ phosphate buffer $\mathrm{pH}$ 7.4, at room temperature. The brain was removed from the skull and post fixed for an additional $1 \mathrm{~h}$ at $4^{\circ} \mathrm{C}$. Slices, $250-\mu \mathrm{m}$ thick, cut on a Vibratome (TPI, St. Louis, MO, USA), were rinsed in phosphate buffer and stored at $-20^{\circ} \mathrm{C}$ for later processing. Cryoprotection was provided by infiltration and storage in a mixture of $20 \%$ glycerin and $2 \%$ dimethyl sulfoxide (DMSO, Merck) in phosphate buffer.

Because antibodies against the vesicular glutamate transporters showed insufficient penetration in standard sections ( $\geq 50 \mu \mathrm{m}$ thick) we resectioned the $250-\mu \mathrm{m}$-thick 
sections into $10-\mu \mathrm{m}$-thin sections prior to starting the immunofluorescence procedure.

Immunofluorescence staining procedure

\section{Double-immunofluorescence staining}

All procedures were carried out with free-floating sections. Sections were rinsed with $50 \mathrm{mM}$ Tris/ $\mathrm{HCl}$ buffer, $\mathrm{pH} 8.0$ containing $0.5 \%$ Triton X-100) (Sigma, St. Louis, MO, USA) and preincubated for $1 \mathrm{~h}$ at room temperature in $5 \%$ normal goat serum (Nordic, Tilburg, The Netherlands) and $2 \%$ bovine serum albumin (BSA, Sigma). Incubation was carried out for $72 \mathrm{~h}$ at $4^{\circ} \mathrm{C}$, under continuous gentle agitation, with a cocktail of mouse anti-CR (1:800; Chemicon, Temecula, CA, USA), and rabbit anti-VGluT1 or rabbit anti-VGluT2 (1:800; both antibodies purchased from Synaptic Systems, Göttingen, Germany) containing 5\% normal goat serum. The secondary antibodies were pooled as well: Alexa Fluor ${ }^{\mathrm{TM}}$ 488-conjugated $F\left(\mathrm{ab}^{\prime}\right) 2$ fragments of goat-anti-mouse IgG (1:100; Invitrogen-Molecular Probes, Eugene, OR, USA) mixed with Alexa Fluor ${ }^{\mathrm{TM}}$ 594-conjugated goat anti-rabbit IgG (1:100; InvitrogenMolecular Probes).

\section{Triple-immunofluorescence staining}

Sections were preincubated for $1 \mathrm{~h}$ at room temperature with $5 \%$ normal donkey serum containing $0.3 \%$ Triton $\mathrm{X}$ 100. Incubation was overnight at room temperature and under continuous gentle agitation with a cocktail of rabbit anti-VGluT2 (1:500 in blocking solution; Synaptic Systems), sheep anti-GAD (1:400; gift by Dr. Wolfgang Oertel, University of Munich, Germany, characterized by Oertel et al. 1981), and mouse anti-CR (1:250; Chemicon). Immunoreactivities were visualized via incubation with a cocktail of $\mathrm{Cy} 2^{\mathrm{TM}}$-conjugated donkey anti-goat $\mathrm{IgG}$ (1:400; Dianova, Hamburg, Germany), Сy $3^{\mathrm{TM}}$-conjugated donkey anti-rabbit $\mathrm{IgG}$ (Dianova) and $\mathrm{Cy} 5^{\mathrm{TM}}$-conjugated donkey anti-mouse IgG (Dianova). After incubation, the sections were extensively rinsed with buffer, transferred to distilled water, mounted on glass slides, dried and coverslipped with Entellan (Merck).

\section{Control incubations}

We took measures to ensure that the observed fluorescence could be attributed to its proper marker only. Possible false-positive immunostaining was checked by means of a series of incubations with: (a) one of the primary antibodies instead of the cocktail but with the complete cocktail of fluorochromated secondary antibodies, (b) single, pairs or triplets of the primary antibodies followed up by several combinations of fluorochromated antibodies in order to notice cross-species immunostaining, (c) "wrong" combinations of single primary antibody and single fluorochromated secondary antibody, (d) a cocktail of primary antibodies followed by incubation with buffer only, (e) buffer only in lieu of primary antibodies followed by incubation with the complete cocktail of secondary antibodies.

Inspection of these sections strengthened our notion that the staining of each antibody was specific and that there was no interference. Sections treated with the "wrong" combinations of antibodies were blank. Cross-species fluorescence was absent. Staining of structures with each of the VGluT antibodies was limited to punctae, small structures with shapes and sizes in the same range as axon terminals and fibers expanding into axon terminals. Immunostaining with the anti-GAD antibodies revealed a punctate pattern of fiber terminals similar to that observed already long ago by many authors, including us (Oertel et al. 1981, 1982; Wouterlood et al. 1985). Sections immunostained with the anti-CR antibodies contained well-stained, complete neurons, including cell bodies, dendrites, fibers and axon terminals, matching previous findings (Wouterlood et al. 2000).

Single, double and triple-label confocal laser scanning microscopy

Sections were initially analyzed in Leipzig with a Zeiss LSM 510 Meta confocal laser scanning microscope (CLSM) equipped with the laser excitation lines 488, 568 and $633 \mathrm{~nm}$ (Carl Zeiss, Oberkochen, Germany). In Amsterdam we first studied sections in a Leica TCS-SP confocal instrument equipped with an Argon/Krypton gas laser (Leica Microsystems, Heidelberg, Germany): 488, 568 and $647 \mathrm{~nm}$ excitation wavelengths, and ultimately in a Leica TCS-SP2-AOBS instrument equipped with an Argon/Krypton gas laser (488 nm) and with HeNe lasers supplying excitation at 543 and $633 \mathrm{~nm}$. Irrespective of the instrument, we configured two or three analysis channels. A channel is a specific configuration of the CLSM designed to detect a particular fluorochrome: laser, emission wavelength, dichroic mirror, filter set and detector. Next, small regions of interest were viewed at high magnification with a high-resolution objective lens (Leipzig: $63 \times$ oil, NA 1.40; Amsterdam: 63× water NA 1.2 (TCS-SP) and $63 \times$ glycerin, NA 1.30 (TCS-SP2). Finally, double- or triple-channel series of images were obtained through the $63 \times$ immersion lens at high zoom setting (zoom 8 , pinhole 
1.00 arbitrary units [Airy disk] 100\%, Z-increment always $122 \mathrm{~nm}, 40-100$ images of $512 \times 512$ pixels at 8-bit sampling). Additional measures at image acquisition time to suppress crosstalk were the following. First, we always acquired images in sequential mode, exciting each fluorochrome with its own specific laser light. We scanned also single-stained sections and sections containing single structures (axons labeled with a neuroanatomical tracer) triple-stained in order to determine whether the instrument settings were correct, that is that signal separation was unconditional.

The sampling scheme included two or three samples per cortical layer per section (see Fig. 1a, b). We carefully avoided at this point cell bodies and capillaries because these would interfere with quantification. In total we scanned the medial subdivision of the entorhinal cortex (MEA) in six horizontally cut sections, and the lateral subdivision of the entorhinal cortex (LEA) in six coronally cut sections. In each acquisition session, we took for calibration and alignment control purposes images of the triple-stained control sections mentioned above, using unchanged instrument parameters.

Post acquisition computer processing

\section{Deconvolution}

All image files were processed with Huygens Professional deconvolution software (Scientific Volume Imaging, Hilversum, The Netherlands) running on a Silicon Graphics Origin $300^{\mathrm{TM}}$ computer. Deconvolution is necessary at this ultrahigh magnification to extract in a statistically reliable way all relevant information from the image series and to reduce blur and background (van der Voort and Strasters 1995; Wouterlood et al. 2002, 2007; Wouterlood 2006). Figure 5a, b shows an image before ("raw" image) and after deconvolution. The images obtained from the triplestained calibration sections were used to determine whether and to what degree correction might be necessary for pixel shift between channels caused by errors like laser unalignment or chromatic aberration.

\section{Threshold analysis and 3D object recognition with ImageJ}

Immunostaining with the antibodies against VGluTs and GAD typically produced punctate-staining patterns. Small aggregates of fluorescence seen by the observer's eye ("punctae") are translated by digital imaging into clumps of pixels with elevated grey intensities. Output of a CLSM consists of multichannel Z-series of images in which each frame is a $2 \mathrm{D}$ bitmapped image containing islands of pixels
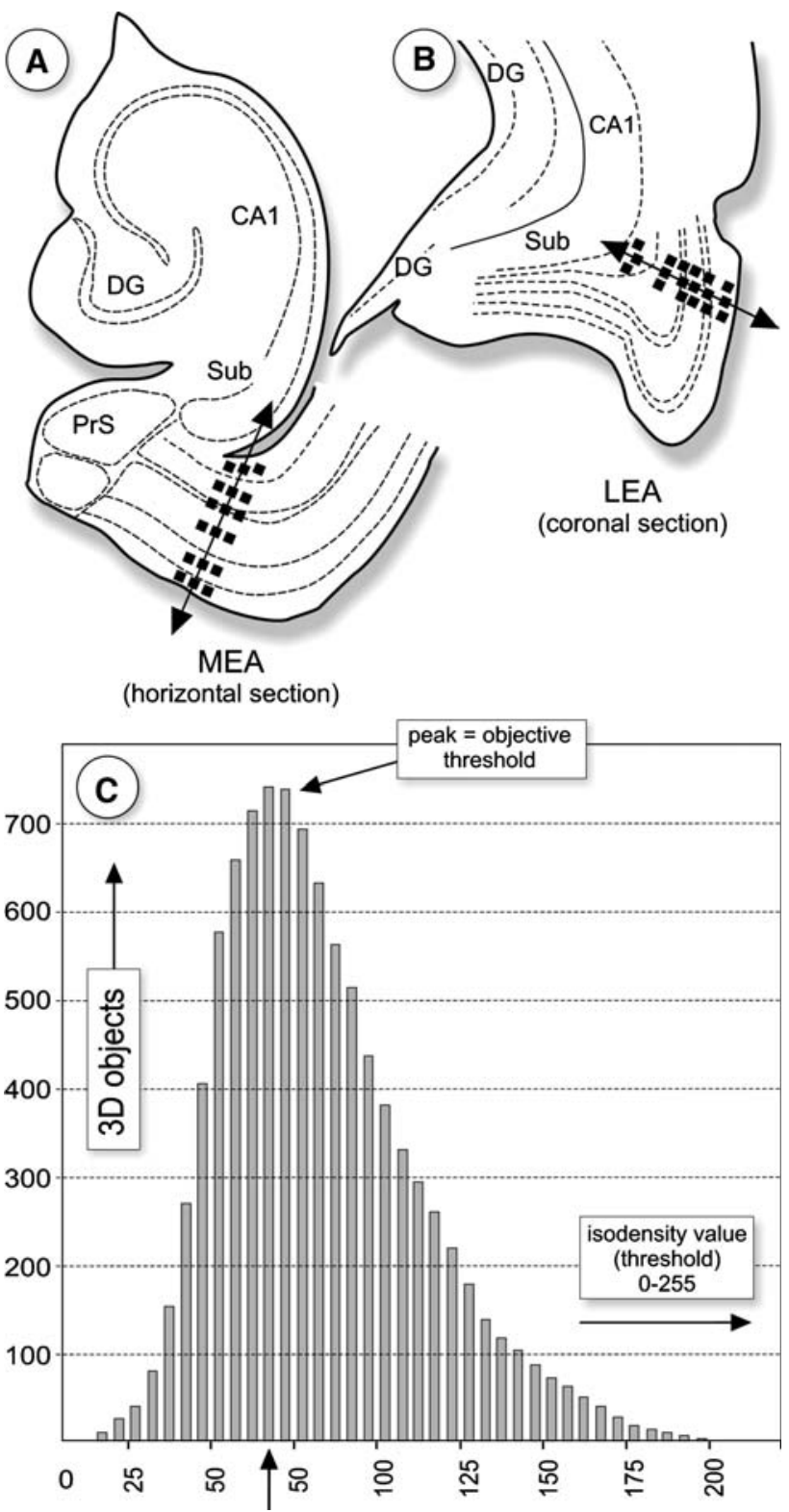

Fig. 1 Sampling scheme: three samples per layer, all layers of the entorhinal cortex. MEA was sampled in horizontal sections, LEA in frontal sections. $D G$ dentate gyrus, $S u b$ subiculum, $\operatorname{Pr} S$ presubiculum. $C$ Intensity-3D object histogram calculated by the ImageJ 3D object recognition plugin: number of $3 \mathrm{D}$ objects ( $Y$-axis) plotted against threshold isodensity value ( $X$-axis). For each sample we determined this histogram and used the intensity causing the peak of this histogram as the "objective" threshold further used to count 3D objects

that represent the "optically sectioned" small aggregates of fluorescence. Programs aimed at 3D object recognition attempt the reverse: they identify islands of pixels with elevated intensities in each frame of a $Z$-series by wrapping them in isodensity "envelopes". Next, successive frames in the $Z$-series are compared and the isodensity envelopes are extended into the Z-direction. In the present paper, we use the term " $3 \mathrm{D}$ object" where we deal with the results of the 
digital imaging, 3D object recognition and 3D reconstruction.

From the above it must be clear that $3 \mathrm{D}$ object recognition hinges on objective and reproducible thresholding of the isodensity envelopes. Thus we started with threshold analysis of all image series, using ImageJ, a public domain, Java-based image analysis suite (Rasband 1997-2006). Specifically, we applied the plugin "3D object counter" (Fabrice Cordelières, Institut Curie, Orsay, France; fabrice.cordelieres@curie.u-psud.fr). This plugin wraps all relevant punctae present in a $Z$-series with isodensity envelopes and counts their numbers ("3D objects"). We applied a macro written by Dr. Riichi Kajiwara (Neuroscience Research Institute, AIST, Tsukuba, Japan) that runs the plugin through the entire range of isodensity intensity thresholds (0-255). Typical for threshold analysis of punctate immunofluorescence is that the number of recognized 3D objects plotted against threshold intensity reveals a parabolic curve (see Fig. 1c). The intensity associated with the maximum number of recognized 3D objects was considered by us to represent the "objective threshold" to be used in all further processing. An offset of 100 voxels was applied to remove noise. Since the absolute number of recognized $3 \mathrm{D}$ objects is a function of the scanned tissue volume we applied a correction by dividing in each sample the number of identified 3D objects by the sample's volume and normalizing the calculated number to a $1,000 \mu \mathrm{m}^{3}$ tissue volume. The outcome of this exercise is further called the number of 3D objects in the "density reference frame" (DRF). We did not correct for tissue shrinkage.

\section{Coexpression of markers}

We processed the deconvoluted confocal image series through software written in SCIL_Image, a C++ implementation (TNO, Delft, The Netherlands). The software includes instructions to detect 3D objects (comparable to the $3 \mathrm{D}$ object counter plugin of ImageJ) and it produces numerical and graphical output with respect to 3D objects found at identical positions in adjacent channels (i.e., punctae in which colocalization occurs). Scripts pass parameters, e.g. thresholds, to the software. The concept and application of these scripts are described in detail in a separate paper (Wouterlood et al. 2007a). As thresholds for the $3 \mathrm{D}$ object recognition in SCIL_Image we used the objective thresholds obtained via the Image $\mathrm{J}$ threshold analysis described above. Because bitmapped images of small different biological objects lying next to each other (apposition) may show overlap due to Abbe diffraction (see Wouterlood et al. 2007a), we considered coexpression of markers to exist only if the involved 3D objects showed at least 500 voxels overlap between channels. A geometrical sphere of 500 voxels in our Z-image series (radius $0.365 \mu \mathrm{m}$ ) roughly corresponds with that of a small axon terminal. In addition to this criterion we ran the entire collected dataset with overlap criteria 100 voxels and 1,000 voxels, and we comment on the findings with these lowered and raised overlap criteria.

\section{$3 D$ reconstruction through surface rendering}

Structures included in the image files were 3D reconstructed by means of Amira ${ }^{\mathrm{TM}}$ visualization/modeling software (developed by the Konrad Zuse Zentrum for Information Technology, Berlin, Germany: website at http://amira.zib.de; program distribution by http://www. amiravis.com). This program creates multichannel surface rendered 3D reconstructions from series of bitmapped images via isodensity wireframes. Thresholding is interactive. We used in Amira the "objective" thresholds determined via the ImageJ threshold analysis.

\section{Results}

Distribution of markers

\section{VGluT1}

At first inspection the immunofluorescence signal in the entorhinal cortex associated with VGluT1 was very strong and even. This, and the uniformity of the stained structural elements, gave the sections at low magnification a "flat" appearance (see Fig. 2a). There were no qualitative differences in this respect between the medial (MEA) and lateral (LEA) subdivisions of the entorhinal cortex. At high magnification and in the confocal laser scanning microscope, VGluT1-immunofluorescence resolved into a distinct punctate pattern (Fig. 2b, c). The punctae, and the corresponding 3D objects after computer reconstruction, were very small (diameter in the order of magnitude of $0.5 \mu \mathrm{m})$, and they were densely packed in the molecular layers and in the neuropil surrounding (completely immunonegative) cell bodies. 3D objects consisted of fiber-like structures and small aggregates of fluorescence matter with shapes and sizes resembling those of axon terminals. We did not see any immunopositive cell body, dendrite or long fiber. Analysis of the sampled areas with the 3D object recognition plugin of ImageJ revealed that the average 3D object, under standardized microscopy conditions with objective thresholding, consisted of 909 voxels on average, with quite some spread reflecting the large variation in dimensions (standard deviation 1,596 voxels; $n=38,862$ 


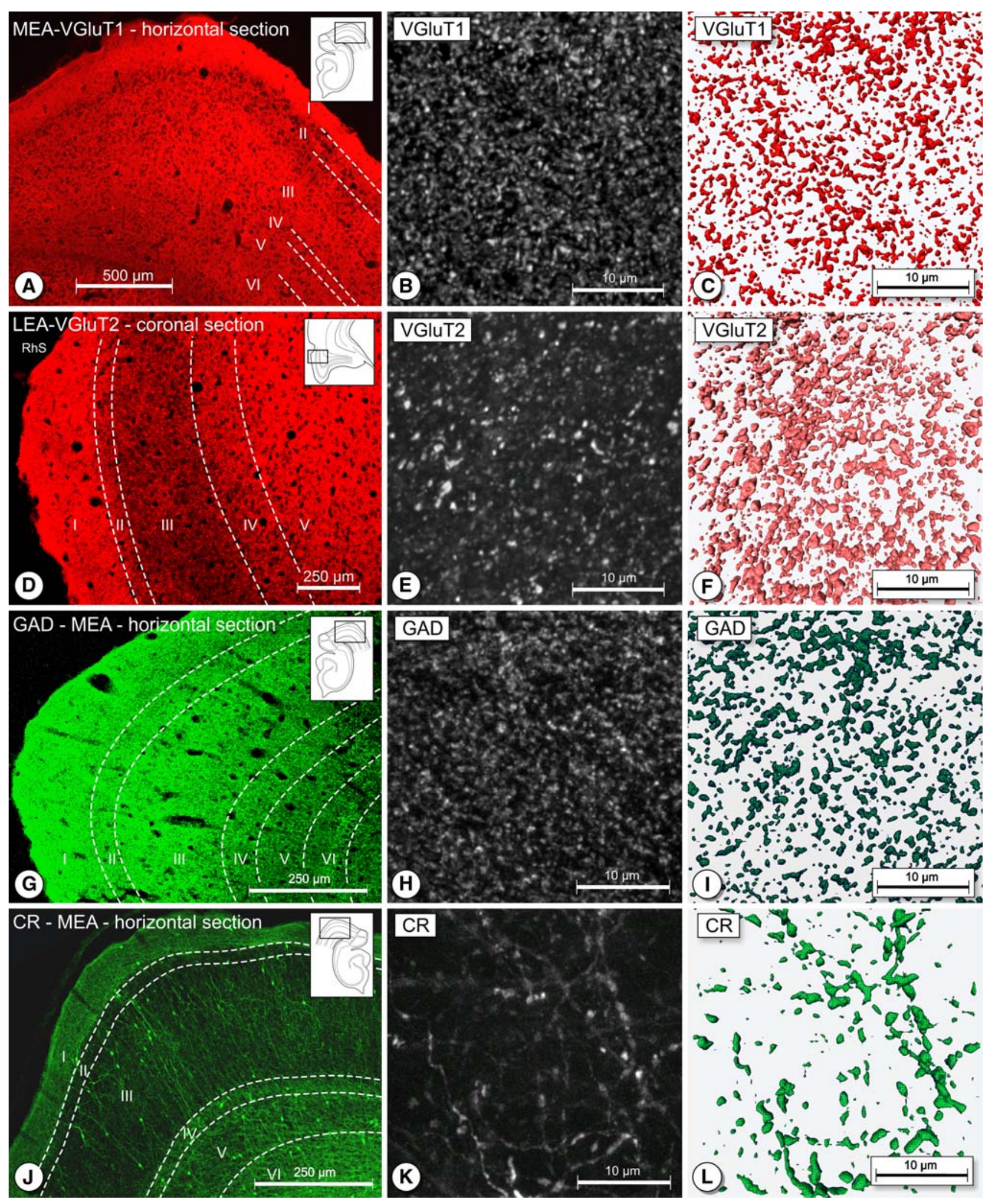

3D objects, smallest 3D object 100 voxels, largest 50,079 voxels). A spherical cluster of 909 voxels has a radius of 6.01 voxels, which fits a box with the dimensions
$12 \times 12 \times 12$ pixels, or in absolute measures a volume enclosed by a box $0.68 \mu \mathrm{m}$ long and wide and, because of $Z$-stepping, $1.46 \mu \mathrm{m}$ deep. In the systematic analysis of the 
४Fig. 2 Distribution of VGluT1, VGluT2, GAD and CR in the entorhinal cortex. a, d, $\mathbf{g}, \mathbf{j}$ : Low-power micrographs showing the typical uniform and dense, yet different distributions of VGluT1-, VGluT2-, GAD- and CR-immunofluorescence, respectively. Cortical layers are indicated with dashed lines and accompanying Roman numerals. The insets show the regions where samples were taken for confocal scanning. $\mathbf{b}, \mathbf{e}, \mathbf{h}, \mathbf{k}$ High magnification projection images of Z-image series of VGluT1-, VGluT2-, GAD- and CR punctae, sampled in layer I of LEA $(\times 63$ magnification, $\times 8$ zoom, after deconvolution). c, f, i Corresponding $3 \mathrm{D}$ reconstructions of $3 \mathrm{D}$ objects, K. 3D reconstruction of CR fibers. $R h S$ rhinal sulcus

density of 3D objects through all cortical layers we found in layer III of MEA the densest distribution of VGluT1 punctae expressed as the number of $3 \mathrm{D}$ objects in the $\left(1,000 \mu \mathrm{m}^{3}\right) \mathrm{DRF}(140 \pm 36$; see Table 1$)$. For LEA we observed the highest density of VGluT1 punctae in layer III as well, i.e. $112 \pm 83 \mathrm{D}$ objects in the DRF. All layers taken together, the average density in MEA amounted to 123 3D objects in the DRF versus 104 in LEA. Thus, VGluT1 appeared to be most ubiquitous in MEA. All data taken together showed 113 VGluT1 3D objects in the "average" entorhinal DRF.

\section{VGluT2}

The distribution of fluorescence label after VGlut2 immunostaining was in several aspects different from that of VGluT1. Initial visual inspection at low magnification indicated fluorescence throughout all layers (Fig. 2d). At high magnification, VGluT2 immunostaining showed a punctate aspect with small aggregates of fluorescence (Fig. 2e, f) and with conspicuous absence of fluorescence signal in cell bodies and dendrites. However, individual VGluT2 punctae seemed larger than the punctae seen after VGluT1 immunostaining and they seemed less densely distributed. Visual comparison of MEA and LEA did not reveal major differences. Moderate amounts of VGluT2 punctae appeared in all layers in both cortical subdivisions, with many punctae in layers III-IV. Typical for layers II and III were dense deposits of fluorescence punctae in the neuropil in between the clusters of completely immunonegative cell bodies.

ImageJ analysis revealed that the average number of voxels making up one aggregate of VGluT2 immunofluorescence was 1,777 (SD 4,493; $n=33,068$, smallest 3D object 100 voxels, largest 98,622 voxels). A spherical cluster of voxels of the average size has a radius of 7.51 voxels, fitting the inside of a box measuring $15 \times 15 \times 15$ pixels, or in absolute measures a box $0.87 \mu \mathrm{m}$ long and wide and, because of $Z$-stepping, $1.83 \mu \mathrm{m}$ deep.

Quantitatively, VGluT2 3D objects appeared unevenly distributed throughout the cortical layers, their numbers varying between 53 and $843 \mathrm{D}$ objects in the DRF. We noted in this respect slight differences between MEA and LEA (Table 1). The density of 3D objects in layer I in both MEA and LEA was moderate. Layers II and III of MEA showed considerably higher densities of 3D objects than the corresponding layers in LEA. Layer III showed both in MEA and LEA a higher density of 3D objects than layers I and II with, of both subdivisions, MEA expressing the highest density (layer II: $79 \pm 5$, layer III: $80 \pm 8$ in the DRF). Layers III and IV formed a continuum in LEA and could not be further differentiated. In contrast with LEA, layer IV was readily distinguishable in MEA and of the entire entorhinal cortex this layer contained the highest density of 3D objects in the DRF: $84 \pm 11$. Layers V and VI in both MEA and LEA contained moderate numbers of VGluT2 3D objects. Layer VI in MEA had the lowest density of the entire entorhinal cortex, notably $53 \pm 103 \mathrm{D}$ objects per DRF. All layers lumped together, the average density of VGluT2 punctae in

Table 1 Quantification of punctae immunopositive for VGluTs and GAD

\begin{tabular}{|c|c|c|c|c|c|c|}
\hline \multirow[t]{2}{*}{ Layer } & \multicolumn{3}{|c|}{ MEA/number of $3 \mathrm{D}$ objects } & \multicolumn{3}{|c|}{ LEA/number of 3D objects } \\
\hline & VGluT1 & VGluT2 & GAD & VGluT1 & VGluT2 & GAD \\
\hline I & $129 \pm 12$ & $61 \pm 8$ & $108 \pm 33$ & $102 \pm 18$ & $62 \pm 5$ & $118 \pm 28$ \\
\hline II & $128 \pm 17$ & $79 \pm 5$ & $86 \pm 35$ & $92 \pm 15$ & $58 \pm 7$ & $97 \pm 14$ \\
\hline III & $140 \pm 36$ & $80 \pm 8$ & $74 \pm 15$ & $112 \pm 8$ & $73 \pm 10$ & $88 \pm 18$ \\
\hline IV & $121 \pm 15$ & $84 \pm 11$ & $66 \pm 17$ & $103 \pm 12$ & $\mathrm{NC}$ & $103 \pm 31$ \\
\hline V & $118 \pm 11$ & $55 \pm 8$ & $68 \pm 8$ & $104 \pm 12$ & $71 \pm 4$ & $93 \pm 22$ \\
\hline VI & $104 \pm 6$ & $53 \pm 10$ & $84 \pm 35$ & $113 \pm 2$ & $62 \pm 8$ & $108 \pm 32$ \\
\hline DRF average & 123 & 68 & 82 & 104 & 65 & 101 \\
\hline VGluT1 + VGluT2 + GAD 3D objects in the DRF & 273 & & & 270 & & \\
\hline
\end{tabular}

Data of each cortical layer is the average of six samples. Numbers obtained with ImageJ, 3D object counter plugin, using the "objective" threshold. Calculated is the number of 3D objects normalized per $1,000 \mu \mathrm{m}^{3}$ density reference frame (DRF). $n c$ not counted (because we could not distinguish layer IV in LEA in the VGluT2 stained material). CR punctae were not counted since CR objects have a quite different form factor than the VGluTs and GAD: it is present in cell bodies and dendrites next to axon terminals 
MEA was 68 3D objects in the DRF whereas in LEA this number was 65 . On average a DRF in the entorhinal cortex contained 67 VGluT2 3D objects.

\section{VGluT1 compared with VGluT2}

Initial visual comparison of the VGluT1- and VGluT2immunostained sections gave the impression of a much lower density of VGluT2 punctae compared with VGluT1punctae, especially in layer III (compare Fig. 2a, d). The quantitative approach confirmed this observation. Expressed as the number of 3D objects in the DRF, VGluT1 3D objects occurred in all immunostained sections at roughly $50 \%$ higher density compared with VGluT2. This higher density of VGluT1 3D objects was observed in sections taken from different rat brains as well as in pairs of adjacent sections of the same rat brain immunoprocessed in parallel with the two different anti-VGluT antibodies. Not only the numbers of VGluT1- or VGluT2 3D objects packed in the same DRF were different, also the sizes of the immunostained punctae differed considerably. The difference in volume between the average VGluT1- and VGluT2 3D-object was 909 against $1,777=868$ voxels. Thus, the average VGluT2 3D object had a diameter 3 voxels larger than that of the average VGluT1 3D object. Statistical testing ( $T$ test, $z$ test) revealed a significant difference in diameter at $P<0.01$.

\section{$G A D$}

In the sections subjected to GAD-immunohistochemistry we noted immunopositive cell bodies next to dense punctate staining representing fiber terminals (cf. Oertel et al. 1982). The immunopositive punctae appeared at first glance evenly distributed throughout the cortical layers (Fig. 2g-i). In the quantitative analysis, expressed as the number of 3D objects in the DRF, layer I in both MEA and LEA had relatively the highest density of GAD 3D objects whereas the lowest densities were encountered in LEA in layer III and in MEA in layer V (Table 1). Taken all layers together, the density of GAD 3D objects was in LEA approximately $20 \%$ higher than in MEA.

Image $J$ analysis revealed that the average number of voxels making up one aggregate of GAD immunofluorescence was 1,242 (SD 2,377; $n=19,157$, smallest 3D object 100 voxels, largest 47,249 voxels). A spherical cluster of voxels of this size has a radius of 6.67 voxels corresponding with a spherical volume fitting roughly the inside of a $13 \times 13 \times 13$ pixel box, or in absolute dimensions a box $0.75 \mu \mathrm{m}$ long and wide, and because of $Z$-stepping size, $1.58 \mu \mathrm{m}$ deep.

\section{Calretinin}

CR-positive cell bodies were scattered in the cortical layers of both subdivisions of the entorhinal cortex (Fig. 2j; MEA). The highest density of CR positive cells occurred in LEA. Cell bodies and processes stood crisply out against the background, at least in the outer layers (I-IV) of EC. Wellstained CR immunopositive cells displayed bipolar and multipolar configurations of their dendritic trees, with long, slender and aspiny dendrites. Cell bodies embedded in layers II and III displayed in several cases dendrites that ascended all the way to the brain surface. In LEA a very dense plexus of CR-positive fibers was present just below the cortical surface. This plexus is typical for LEA and contains calretinin expressing fibers originating in the olfactory bulb (Wouterlood and Härtig 1995). All layers of LEA and MEA contained moderate amounts of CR positive fibers, with the highest density in layer III of LEA in the region adjacent to the perirhinal cortex. In MEA, fibers were generally less densely distributed than in LEA. All these observations were similar to those reported earlier by us (Wouterlood et al. 2000).

Since CR, in contrast to the VGluTs and GAD is expressed in cellular compartments whose dimensions are of another magnitude (cell bodies, dendrites) than those of the VGluT1-, VGluT2- and GAD punctae, we assessed that a quantitative comparison of numbers of identified 3D CR objects in a density reference frame with those of VGluTand GAD 3D objects would not be meaningful. For the sake of completeness, and to enable visual comparison with the VGluTs and GAD we include an image of CR positive axons in Fig. 2k and a 3D reconstruction in Fig. 21.

\section{GAD compared with VGluTs}

GAD 3D objects had a lower density in MEA compared with LEA. The figures for VGluT1 3D objects were the reverse (except in layer VI where VGluT1 3D objects had the highest density in LEA). On average, the distribution of VGluT2 3D objects was equally dense in MEA and LEA. Although the proportion of GAD 3D objects to VGluT1 3D objects differed between MEA and LEA, the combined densities of VGluT1- and GAD 3D objects in the $1,000 \pm \mathrm{m}^{3}$ DRF was in both subdivisions equal to 205 . Numbers of VGluT2 punctae in the DRF did not differ significantly between MEA and LEA (68 in MEA, 65 in LEA). All data on VGluT1-, VGluT2- and GAD 3D objects together show that a $1,000 \mu^{3}$ DRF in MEA hosts 273 3D objects while the comparable DRF in LEA hosts 270 3D objects. Thus, seen in the perspective of the total population of 3D objects in a DRF, in MEA the density of VGluT1 punctae was higher than in LEA at the relative expense of the density of GAD 3D objects (Fig. 3). 
The average VGluT1 3D object contained 909 voxels, the average VGluT2 3D object contained 1,777 voxels, whereas the average GAD 3D object was composed of 1,242 voxels. In this respect an average GAD 3D object was smaller than an average VGluT2 3D object but bigger than an average VGluT1 3D object.

\section{Coexpression of markers}

\section{VGluT1 and calretinin}

We found only sporadic coexpression, almost nihil, of VGluT1 and CR.

\section{VGluT2 and calretinin}

Coexpression of VGluT2 and CR occurred in all layers, both in MEA and LEA. 3D objects coexpressing both markers were present in all inspected sections and in all samples (Figs. 4, 5). Normalized numbers of 3D objects expressing each single marker, and 3D objects expressing both markers and their percentages are shown in Table 2 . In MEA the highest number of 3D objects coexpressing VGluT2 and CR was present in layer VI (22 3D objects coexpressing VGluT2 and CR on a total of 85 VGluT2 3D objects in the DRF, or 26\%). In LEA the highest number of $3 \mathrm{D}$ objects coexpressing both markers was seen in layer IV (6 3D objects coexpressing VGluT2 and CR on 41 VGluT2 $3 \mathrm{D}$ objects in the DRF). The overall percentage of VGluT2

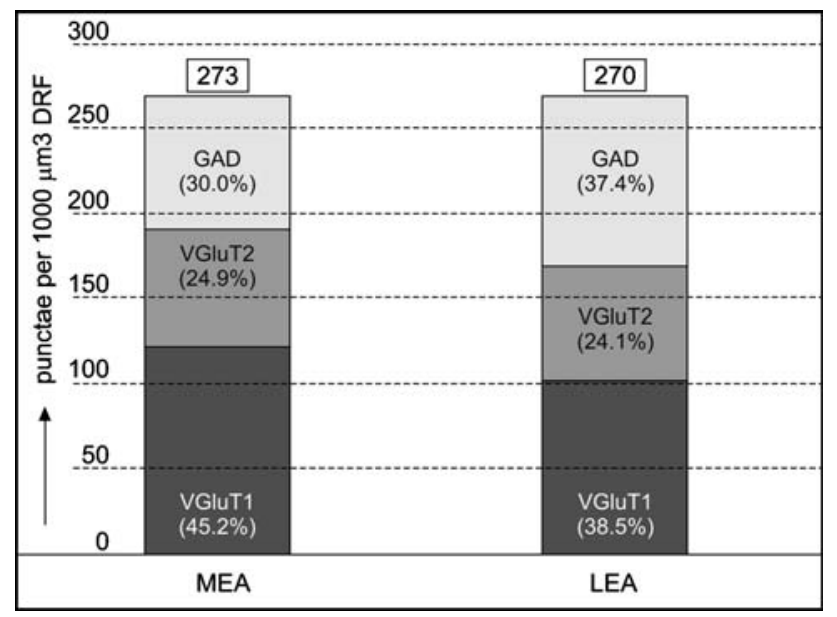

Fig. 3 Absolute and relative distributions of VGluT1, VGluT2 and GAD punctae in MEA and LEA. Data expressed in absolute and relative ways. The ordinate indicates the absolute number of punctae counted in the $1,000 \mu \mathrm{m}^{3}$ DRF: these are average DRF's with contributions of all cortical layers. The sum of the number of VGluT1, VGluT2 and GAD punctae in MEA equals that in LEA. Within each column the percentage of punctae of each contributing marker is represented as the fraction of the sum of all punctae
3D objects, which coexpressed CR, was in MEA $23 \%$ and in LEA $20 \%$. For the entire entorhinal cortex, $12 \%$ of the VGluT2 immunopositive 3D objects also expressed CR.

Percentages of coexpression were also calculated for the calretinin 3D objects. In MEA, 33\% of the CR immunopositive 3D objects coexpressed VGluT2, and in MEA $16 \%$. These percentages were lower than the percentages of VGluT2 3D objects coexpressing CR.

In addition to using 500 voxels as the limiting criterion for overlap we also programmed SCIL_Image to calculate numbers of 3D objects and percentages of overlap based on a 1,000 voxel criterion. Using this higher criterion the absolute number of recognized 3D objects was less than with the 500 voxel criterion and consequently the absolute number of 3D objects showing coexpression was lower as well. However, the percentages of 3D objects showing coexpression stayed roughly the same as for the 500 voxel criterion. For instance, at the 1,000 voxel criterion, in MEA $24 \%$ of the 3D objects containing VGluT2 also expressed CR (732 VGluT2-CR 3D objects on a total of 3,090 VGluT2 3D objects), and in LEA the corresponding percentage was 11 (302 VGluT2-CR 3D objects on a total of 2,778 VGluT2 3D objects). When the overlap criterion was decreased to 100 voxels, percentages of 3D objects coexpressing both markers changed to $31 \%$ for MEA and $23 \%$ for LEA.

\section{$G A D$ and $C R$}

A fair number of 3D objects expressing GAD contained also CR. Coexpression occurred in all cortical layers and we noted such 3D objects in all samples. Percentages were lower than those for VGluT2-CR coexpression. For instance, SCIL_Image reported in MEA 13\% of the 3D objects expressing GAD also expressing CR (10 coexpressing 3D objects in the DRF versus $75 \mathrm{GAD}$-expressing 3D objects). For LEA the corresponding figure was $12 \%$ (6 coexpressing 3D objects in the DRF versus 49 GAD 3D objects). 3D GAD objects in MEA expressing CR amounted to $24 \%$ of the total number of CR 3D objects. For LEA this figure was $12 \%$. Also in case of GAD-CR coexpression the percentages of overlapping 3D objects did not change much if the overlap criterion was raised to 1,000 voxels or lowered to 100 voxels.

\section{VGluT2 and GAD}

A considerable number of 3D objects contained both VGluT2 and GAD. In MEA, $13 \%$ of the recognized VGluT2 3D objects coexpressed GAD (8 3D objects in the DRF on a total number of 61 VGluT2 3D objects). In LEA 
Table 2 Colocalization determined with SCIL_Image software of markers in 3D objects (VGluT2, GAD, and CR) in triple-immunostained sections

$\begin{array}{llllllll}n \text { VGluT2 } & n \text { GAD } & n \text { CR } & n \text { VGluT2 } & n \text { GAD } & n \text { VGluT2 } & \text { n GAD 3D } & \mathrm{n} \text { 3D objects } \\ \text { 3D objects } & \text { 3D objects } & \text { 3D objects } & \text { 3D objects } & \text { 3D objects } & \text { 3D objects } & \text { objects that } & \text { that coexpress } \\ & & & \text { that coexpress } & \text { that coexpress } & \text { that coexpress } & \text { coexpress } & \text { all three markers } \\ & & & \mathrm{CR} & \mathrm{CR} & \text { GAD } & \text { VGluT2 } & \end{array}$

\section{MEA}

$\begin{array}{llll}\text { Layer I } & 49 & 64 & 50 \\ \text { Layer II } & 50 & 89 & 44 \\ \text { Layer III } & 65 & 75 & 39 \\ \text { Layer IV } & 55 & 72 & 31 \\ \text { Layer V } & 62 & 63 & 44 \\ \text { Layer VI } & 85 & 86 & 46 \\ \text { Total MEA } & \mathbf{6 1} & \mathbf{7 5} & \mathbf{4 2} \\ \text { LEA } & & & \\ \text { Layer I } & 49 & 54 & 62 \\ \text { Layer II } & 42 & 48 & 48 \\ \text { Layer III } & 35 & 47 & 43 \\ \text { Layer IV } & 41 & 52 & 58 \\ \text { Layer V } & 34 & 44 & 44 \\ \text { Layer VI } & 39 & 49 & 48 \\ \text { Total LEA } & \mathbf{4 0} & \mathbf{4 9} & \mathbf{5 0} \\ \text { LEA + MEA } & 50 & 62 & 46\end{array}$

$\begin{array}{lllll}10(20 \%) & 7(11 \%) & 6(12 \%) & 6(9 \%) & 1 \\ 13(26 \%) & 9(10 \%) & 5(10 \%) & 6(7 \%) & 1 \\ 17(26 \%) & 12(16 \%) & 9(14 \%) & 10(13 \%) & 1 \\ 11(20 \%) & 6(8 \%) & 3(5 \%) & 4(6 \%) & 1 \\ 13(21 \%) & 9(14 \%) & 8(13 \%) & 9(14 \%) & 1 \\ 22(26 \%) & 17(20 \%) & 14(16 \%) & 9(10 \%) & 2 \\ \mathbf{1 4}(\mathbf{2 3 \%}) & \mathbf{1 0}(\mathbf{1 3 \%}) & \mathbf{8}(\mathbf{1 3 \%}) & \mathbf{5}(\mathbf{7 \%}) & \mathbf{1} \\ & & & & \\ 4(8 \%) & 4(7 \%) & 10(20 \%) & 10(19 \%) & 0 \\ 5(12 \%) & 6(13 \%) & 9(21 \%) & 11(23 \%) & 1 \\ 5(14 \%) & 8(17 \%) & 11(31 \%) & 17(36 \%) & 1 \\ 6(15 \%) & 4(8 \%) & 14(34 \%) & 22(42 \%) & 1 \\ 5(15 \%) & 4(9 \%) & 12(35 \%) & 14(32 \%) & 1 \\ 5(13 \%) & 8(16 \%) & 18(46 \%) & 16(33 \%) & 0 \\ \mathbf{8 ( 2 0 \% )} & \mathbf{6 ( 1 2 \% )} & \mathbf{1 2}(\mathbf{3 0 \% )} & \mathbf{1 5}(\mathbf{3 1 \%}) & \mathbf{1} \\ 6(12 \%) & 6(26 \%) & 10(20 \%) & 10(16 \%) & 1\end{array}$

Normalized numbers of 3D objects (per 1,000 $\mu^{3}$ DRF) and averaged per layer. Minimum 3D overlapping object size was 500 voxels, that is that $3 \mathrm{D}$ objects coexpressing the markers and passing the test shared a common volume of at least 500 voxels. Data include 48 samples in LEA and 33 samples in MEA

the corresponding percentage was more than twice as high: $30 \%$ (12 coexpressing 3D objects on 40 VGluT2 3D objects). Conversely, in MEA, $7 \%$ of the recognized GAD 3D objects expressed VGluT2 (5 3D objects in the DRF expressing both markers on 75 GAD 3D objects). In LEA the corresponding percentage was much higher. In this cortical subdivision the program calculated $31 \%$ of the GAD 3D objects fulfilling the overlap criterion (15 3D objects in the DRF expressing both markers on 49 GAD 3D objects). In entorhinal cortex as a whole, $20 \%$ of the VGluT2 3D objects coexpressed GAD while $16 \%$ of the GAD 3D objects also expressed VGluT2.

After raising the overlap criterion to 1,000 voxels the program still detected scores of $3 \mathrm{D}$ objects coexpressing both markers: for the entire entorhinal cortex, $19 \%$ of the VGluT2 3D objects coexpressed GAD (or conversely, 18\% of the GAD 3D objects coexpressed VGluT2). In absolute numbers: the program distinguished 1,125 coexpressing $3 \mathrm{D}$ objects on a total of 5,868 VGluT2 3D objects and 6,062 GAD 3D objects. If the overlap criterion was lowered to 100 , the coexpression percentages for the entire entorhinal cortex were even higher $(29 \%$ of GAD 3D objects also expressing VGluT2, or $32 \%$ of the VGluT2 objects expressing also GAD). An illustration of VGluT2-GAD coexpressing 3D objects is provided in Fig. $5 \mathrm{f}$.

\section{Triple expression: VGluT2, GAD and calretinin}

Mostly in MEA and occasionally in LEA we encountered $3 \mathrm{D}$ objects in exactly the same location in all three channels, i.e., objects expressing all three markers (Table 2, further elaborated in Table 3, illustrated in Fig. $5 \mathrm{~g}$ ). In absolute terms, the SCIL_Image routines recognized in our samples in total 258 3D objects fulfilling 500 voxels overlap for all three channels, on a total of 8,351VGluT2-, 8,808 GAD- and 7,606 CR 3D objects (numbers found in MEA and LEA combined). The percentage of 3D objects showing triple overlap thus amounted to around $3 \%$ of the numbers of 3D objects exhibiting the individual markers. Expressed as percentages of the numbers of 3D objects in the DRF, it appeared that $1 \%$ of the VGluT2 3D objects, $1 \%$ of the GAD 3D objects and $2 \%$ of the CR 3D objects also expressed the other two markers.

Using the 100 voxel overlap criterion our program detected in total 18,445 GAD-, 16,137 VGluT2- and 15,810 CR 3D objects. The number of 3D objects that showed tripleexpression at this overlap criterion setting amounted to 546 in MEA and 905 in LEA, which is approximately $10 \%$ of the 3D objects containing one of the individual markers. At 1,000 voxel overlap, SCIL_Image still detected 3D objects expressing all three markers, both in MEA and in LEA: 117 
Table 3 Percentages of coexpression and triple expression of VGluT2, GAD and CR in 3D objects in medial (MEA) and lateral (LEA) entorhinal cortex. Analysis with SCIL_Image

\begin{tabular}{|c|c|c|c|c|c|c|c|c|c|}
\hline & \multicolumn{2}{|c|}{$\begin{array}{l}\text { VGluT2-CR } \\
\text { coexpressing } \\
\text { punctae }\end{array}$} & \multicolumn{2}{|c|}{$\begin{array}{l}\text { GAD-CR } \\
\text { coexpressing } \\
\text { punctae }\end{array}$} & \multicolumn{2}{|c|}{$\begin{array}{l}\text { VGluT2-GAD } \\
\text { coexpressing } \\
\text { punctae }\end{array}$} & \multicolumn{3}{|c|}{$\begin{array}{l}\text { Punctae expressing } \\
\text { all three markers }\end{array}$} \\
\hline & $\begin{array}{l}\text { As \% of } \\
\text { number of } \\
\text { VGluT2 - } \\
\text { punctae }\end{array}$ & $\begin{array}{l}\text { As \% of } \\
\text { number } \\
\text { of CR- } \\
\text { objects }\end{array}$ & $\begin{array}{l}\text { As } \% \text { of } \\
\text { number of } \\
\text { GAD- } \\
\text { punctae }\end{array}$ & $\begin{array}{l}\text { As \% of } \\
\text { number of } \\
\text { CR- } \\
\text { objects }\end{array}$ & $\begin{array}{l}\text { As \% of } \\
\text { number of } \\
\text { VGluT2- } \\
\text { punctae }\end{array}$ & $\begin{array}{l}\text { As } \% \text { of } \\
\text { number } \\
\text { of GAD- } \\
\text { punctae }\end{array}$ & $\begin{array}{l}\text { As \% of } \\
\text { number of } \\
\text { VGluT2- } \\
\text { punctae }\end{array}$ & $\begin{array}{l}\text { As } \% \text { of } \\
\text { number } \\
\text { of GAD- } \\
\text { punctae }\end{array}$ & $\begin{array}{l}\text { As \% of } \\
\text { number } \\
\text { of CR- } \\
\text { objects }\end{array}$ \\
\hline MEA & 23 & 33 & 13 & 24 & 13 & 11 & 1 & 1 & 2 \\
\hline LEA & 20 & 16 & 12 & 12 & 30 & 25 & 1 & 1 & 2 \\
\hline $\mathrm{LEA}+\mathrm{MEA}$ & 12 & 13 & 26 & 22 & 20 & 16 & 1 & 1 & 2 \\
\hline
\end{tabular}

"triple" 3D objects on a total of 5,868 VGluT2 objects (2\%), 6,062 GAD objects (2\%) and 5,115 CR objects (2\%). Increasing the overlap criterion had some effect on the absolute numbers and percentages of triple-expressing 3D objects but did not completely eliminate the observation of 3D objects expressing all three markers.

\section{Discussion}

\section{General}

VGluT1- and VGluT2 immunostaining of rat entorhinal cortex resulted in intense and, at low magnification, quite homogeneous staining in both medial (MEA) and lateral (LEA) entorhinal subdivisions. Immunofluorescence signal was present in all cortical layers. At high magnification, small, intensely stained punctae were visible with shapes and sizes similar to axon terminals. There were no VGluT1- or VGluT2-immunopositive cell bodies or dendrites. Similar observations have been reported by others (e.g., Härtig et al. 2003; Nanclares-Alonso et al. 2004). At the level of visual inspection, VGluT2 punctae seemed larger and less in number than VGluT1 punctae. These differences were quantitatively corroborated via systematic sampling in a confocal laser scanning instrument followed by 3D object recognition and counting based on an objective thresholding procedure. Automated recognition of 3D objects and automated determination of coexpression made it possible to compare layers and subdivisions in an objective way, to apply numerical criteria for the purpose of determining coexpression of markers, and to apply criteria to distinguish coexpression from diffraction-associated overlap.

\section{Histological considerations}

We made our observations in $10 \mu \mathrm{m}$ thick sections. Reducing section thickness to this size was necessary to circumvent false-negativity associated with insufficient penetration of antibodies into sections. The quality of penetration is diagnosed by acquiring a $Z$-series of confocal images stepping through the entire thickness of a section. Good penetration translates into images with a high signal to noise ratio everywhere along the $Z$-axis; poor penetration exposes itself by a decrease in a section of the signalto-noise ratio along the $Z$ axis to a minimum, slowly returning to the starting level once the half-way point has been passed. In particular the penetration of VGluT antibodies became poor when section thickness exceeded $10 \mu \mathrm{m}$. Figure $4 \mathrm{c}$ illustrates the overall good penetration of the anti-VGluT2 antibody into our material. Also antibodies to GAD may show poor penetration (cf. Freund et al. 1983), but in our $10 \mu \mathrm{m}$ thick sections penetration of this antibody appeared complete. Antibodies against CR were not handicapped by a low penetration rate. Thus, we took care to eliminate a source of false-negative immunostaining.

Instrument-related considerations: punctae, 3D objects and thresholds

Immunofluorescence staining of VGluT1, VGluT2 and GAD revealed punctae or, in optical terms, aggregates of high-immunofluorescence emission. The confocal instrument produces 2D "optical sections" through these punctae, and our software recognizes individual 3D objects by constructing envelopes of isodense voxels in the $X, Y$ and $Z$ planes. Thresholding, that is assigning a particular grey intensity value to the isodensity envelope, is crucial. Too low threshold settings produce few, large 3D particles with clusters of high-intensity voxels connected by bridges of lower-intensity voxels, whereas too high thresholds produce also few, small 3D objects now consisting of only the highest-intensity voxels. The number of recognized 3D particles as well as their size therefore depends typically on the setting of the isodensity 

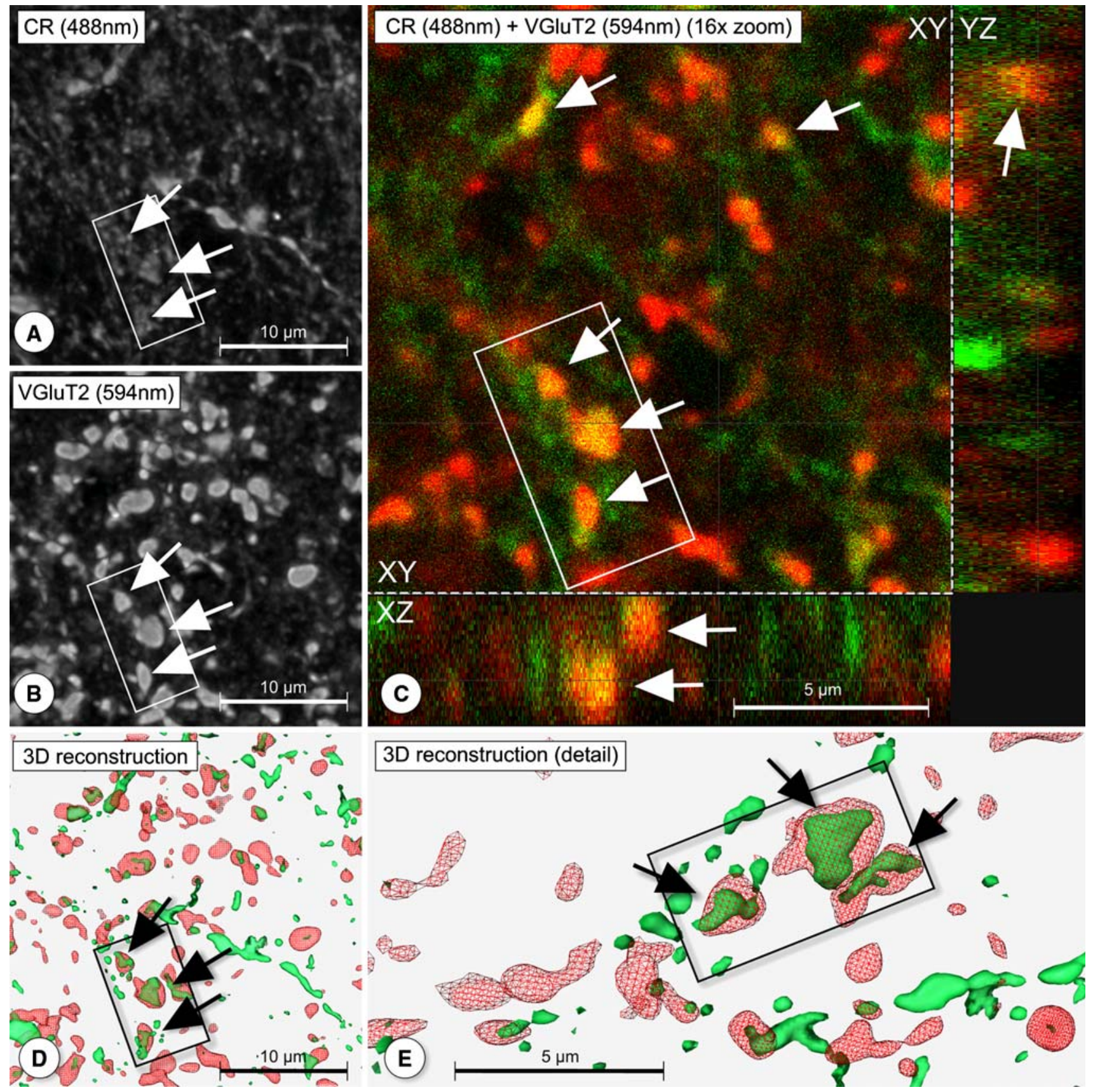

Fig. 4 Coexpression of VGluT2 and CR. This section was doublestained to reveal CR (via Alexa Fluor 488(tm)) and VGluT2 (via Alexa Fluor 594(tm)) d). a Extended-focus view of the $488 \mathrm{~nm}$ channel; distribution of CR. b extended focus view of the companion channel $(594 \mathrm{~nm})$ showing the distribution of VGluT2. c In Section View overlay mode, the $X Y, Z X$ and $Y Z$ images of the 488 and $594 \mathrm{~nm}$ channels are inspected. Green CR, red VGluT2, orange co-localization of signal. d, e computer reconstructions of the structures visible in the $488 \mathrm{~nm}$ channel (green) and $594 \mathrm{~nm}$ channel (red). Punctae coexpressing both markers are indicated with arrows. Image $\mathrm{C}$ was made on purpose for this illustration at zoom $\times 16$. All other imaging at zoom $\times 8$ envelope's threshold. To avoid the strongly subjective and inherently poorly reproducible thresholding by a human computer operator, we opted for applying an operator independent, objective and reproducible thresholding method (Wouterlood et al. 2007a).
Distribution of VGluT- and GAD punctae in entorhinal cortex

VGluT1- and VGluT2 immunoreactive punctae occurred in all layers of the entorhinal cortex. VGluT1 punctae were 

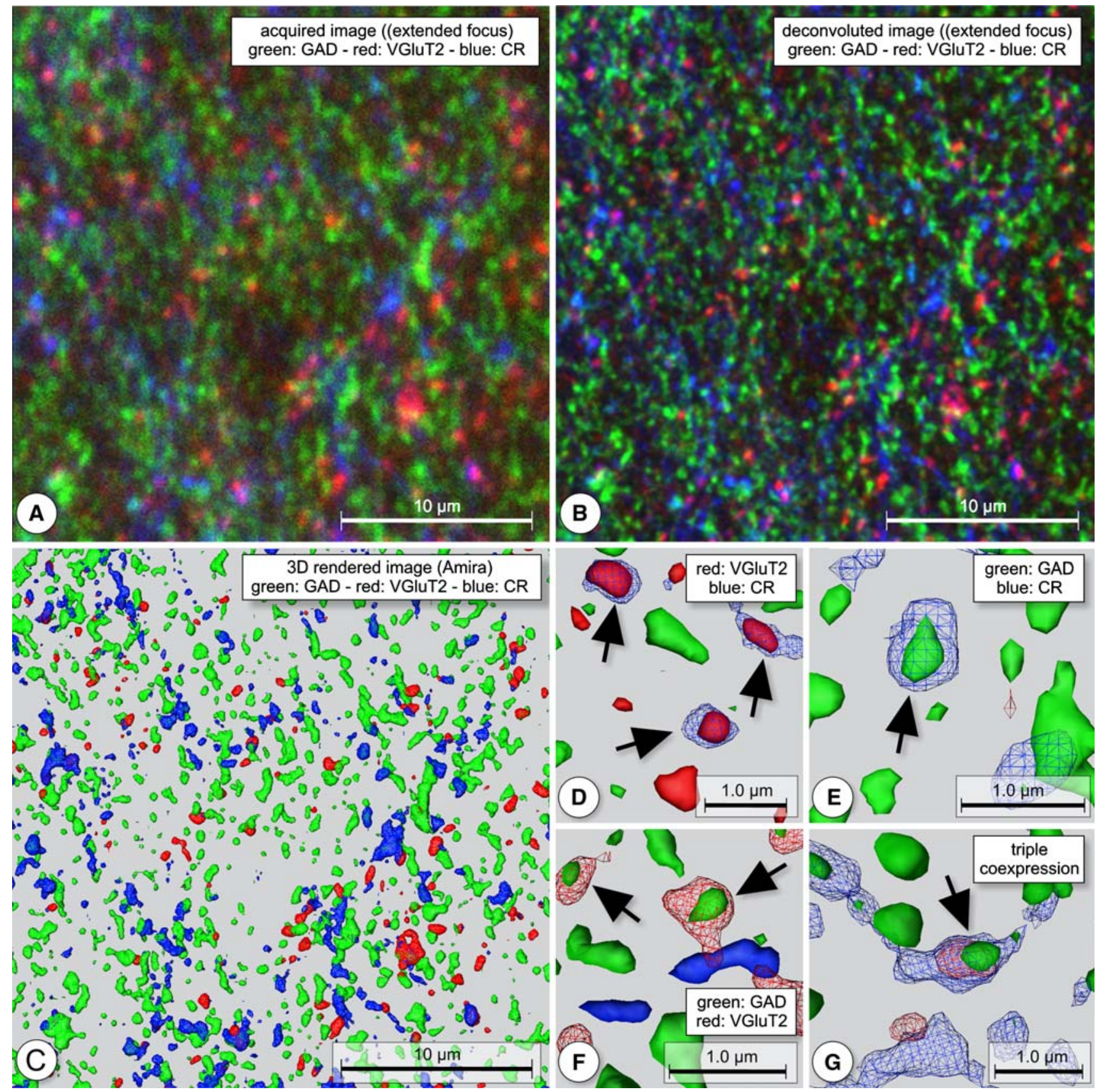

Fig. 5 Triple-immunostained material. Sample B1L1S13S1_ layer02 take_03 (MEA layer III). Colors in all panels: GAD green $(488 \mathrm{~nm})$, VGluT2 red $(543 \mathrm{~nm})$, CR blue $(633 \mathrm{~nm})$. a Extended focus view of the acquired multichannel $Z$-series. b Extended focus image after deconvolution of this image series. c 3D reconstruction.

more abundant than VGluT2 punctae. For each of the VGluTs, going from superficial to deep cortical layers, the normalized densities of immunofluorescent punctae remained fairly constant. Marked differences were found when we compared the VGluT1- and VGluT2 staining patterns. The density of VGluT1 3D objects was considerably and consistently higher than that of VGluT2 3D d-g, Details. d VGluT2 in CR-containing structures (red in blue; arrows). e GAD in CR-containing structures (green in blue; arrow). f GAD in VGluT2-containing structures (green in red; arrows). g All three markers in the same structure (VGluT2, GAD and CR; corresponding colors; structure indicated by an arrow)

objects. At the same time the average VGluT1 3D object was statistically significantly smaller than its VGluT2 counterpart. Because both VGluTs occur presynaptically in axon terminals as components of synaptic vesicle membranes (Bellocchio et al. 1998, 2000: Fremeau et al. 2001), the question arises what underlies the observed differences in size. As the resolving power of a confocal microscope is 
insufficient to distinguish individual synaptic vesicles we must assume that clusters of synaptic vesicles carrying VGluT1 or VGluT2 were imaged. Thus, while the confocal images may provide the contours of clusters of VGluTcarrying synaptic vesicles and thus indicate the presence of axon terminals, these cluster contours may not by default coincide with the limiting membranes of the hosting axon terminals. As a consequence, size differences of immunofluorescent 3D objects must be interpreted in terms of amounts or distributions of clusters of synaptic vesicles. VGluT2 clusters may simply encompass more synaptic vesicles than VGluT1 clusters. Alternatively, with similar size and number of synaptic vesicles, the distances between individual synaptic vesicles in VGluT2 axon terminals, or between small pools of synaptic vesicles, might be larger than in VGluT1 punctae. The electron microscope is necessary to study these possible morphological differences between axon terminals containing VGluT1- or VGluT2 expressing synaptic vesicles.

The distribution of punctae expressing the third marker, GAD, held the middle between that of VGluT1- and VGluT2 punctae. The density of GAD punctae in our samples was homogeneous throughout the different cortical layers.

It is noteworthy that the average densities of VGluT1-, VGluT2- and GAD punctae expressed as the number of 3D objects in the DRF, taken together (VGluT1 + VGluT2 + GAD, Fig. 3) amounted to 273 in MEA and 270 in LEA, which is remarkably similar. In MEA, the DRF density of VGluT1 3D objects was lower than in LEA, but this difference was "compensated for" in MEA by the higher density of GAD 3D objects. One explanation for this phenomenon is that the microarchitecture of brain tissue allows for a fixed total number of axon terminals per tissue volume unit. Within this constraint the tissue volume may be occupied by differently proportioned fractions of axon terminals belonging to specific populations of neurons or projections of different origins. The difference between LEA and MEA may reflect differences in fiber connectivity, architecture and intrinsic wiring (cf. Witter 2002; Wouterlood 2002).

Coexpression in CR 3D objects of VGluT2 and GAD: intrinsic or extrinsic axonal circuits?

Analysis with SCIL_Image revealed that in MEA $33 \%$ of the CR 3D objects coexpressed VGluT2 and that $24 \%$ coexpressed GAD. In LEA the numbers of 3D objects coexpressing either CR and VGluT2 or CR and GAD were half of that in MEA. The overall number in entorhinal cortex of 3D objects coexpressing CR and VGluT2 was $13 \%$. For $3 \mathrm{D}$ objects coexpressing $\mathrm{CR}$ and GAD the similar figure was $22 \%$. Thus we feel that we have solid evidence in support of the hypothesis that there are at least two CR neuron subpopulations in the entorhinal cortex: a GABAergic (GAD-expressing) and a glutamatergic (VGluT2-expressing). Expression of GABA in cell bodies of CR immunopositive neurons in $\mathrm{EC}$ has been reported previously (Wouterlood et al. 2000). The SCIL_Image analysis was not informative about the individual neurons involved since the analysis was limited to punctae, i.e. axon terminals. It might be of interest to determine how many axon terminals belong to the axon collateral distribution of a single CR-VGluT2 neuron and a single CRGAD neuron because such knowledge would enable us to generate models giving more insight in the roles of these neurons in the entorhinal neuronal network.

One important question here remains the origin of the CR-VGluT2 fibers. In a previous study we noted GABAimmunonegative, CR-positive cell bodies embedded in layer I of the entorhinal cortex (Wouterlood et al. 2000). According to the classical Golgi study by Lorente de Nó (1933) layer-I neurons distribute their axon collaterals in layer I only. Therefore, these neurons are considered by us as archetypical interneurons. Subsequently, our hypothesis was that at least these CR-cells might be excitatory. However, the elegant hypothesis exists that VGluT1 is generally associated with cortico-cortical excitatory connectivity and VGluT2 with subcortico-cortical excitatory connectivity (Fujiyama et al. 2001; Kaneko and Fujiyama 2002; reviewed by Fremeau et al. 2004). Thus, we should take the possibility of subcortical CR-glutamatergic afferent innervation of the entorhinal cortex seriously into account.

In the rat, the entorhinal cortex receives fiber projections of subcortical origin. Among these are projections from the medial septum and the diagonal band (Gaykema et al. 1990), i.e., an area known to contain CR-expressing neurons (Résibois and Rogers 1992). Additional subcortical input arises from nuclei of the amygdaloid complex (reviewed by Pitkänen 2000; Kemppainen et al. 2002). Several of these nuclei host neurons that express CR (Résibois and Rogers 1992). A major thalamic input arrives from the nucleus reuniens of the thalamus (NRT; Beckstead 1978, Wouterlood et al. 1990; Vertes et al. 2006) whereas additional diencephalic input is supplied by the paratenial, periventricular and anteromedial thalamic nuclei (Beckstead 1978; van Groen et al. 1995) and the ventromedial hypothalamic nucleus (Canteras et al. 1994). Several of these nuclei contain CR neurons (Résibois and Rogers 1992; Arai 1994). Boutons of paraventricular nucleus fibers ending in the forebrain coexpress $\mathrm{CR}$ and VGluT2 (Härtig et al. 2003). In a follow-up study (Wouterlood et al. 2007b) we report on entorhinal innervation from the NRT. The projection of this nucleus is known in 
detail (Wouterlood et al. 1990; Vertes et al. 2006), its neurophysiology studied in some detail (Dolleman-van der Weel et al. 1997; Bertram and Zhang 1999), and it contains CR immunoreactive neurons (Arai 1994) as well as neurons rich in VGluT2 mRNA (Hur and Zaborszky 2005; Barroso-Chinea et al. 2007).

\section{"Violation" of Dale's postulate: colocalization of VGluT2 and GAD}

Certain findings in the present study, in particular that $20 \%$ of the GAD 3D objects coexpresses VGluT2, contradict what is known as Dale's postulate, "one neuron, one transmitter". We took extreme care to acquire images at nearly ideal channel separation conditions to exclude any trace of false positivity. Yet, at our extremely high magnification, optical diffraction may have induced false positivity. A consequence of Abbe's lens diffraction equation is that image acquisition in two confocal channels of very small and closely apposed biological objects (e.g., axon terminals synapsing with dendritic spines) may lead to 3D reconstructions in which the objects partially overlap (Wouterlood et al. 2007a). For this reason we imposed for coexpression a stringent overlap criterion, rejecting all identified $3 \mathrm{D}$ objects overlapping 500 voxels or less. In spite of this measure, no matter what amount of threshold was applied, we consistently observed considerable numbers of CR 3D objects coexpressing VGluT2 and GAD 3D objects coexpressing VGluT2. Minimally one percent of the 3D objects positively overlapped in all three channels (Figs. 4, 5), no matter the overlap criterion. Thus we feel confident that in the entorhinal cortex small structures really exist expressing both a GABA-marker (GAD) and a glutamate marker (VGluT2). Consistently there are small 3D objects that contain CR plus these two markers. This brings us back to Dale's postulate. This "dogma" has been challenged before, first with the observation of choline acetyltransferase and GABA in amacrine cells in the retina (Brecha et al. 1988), later by evidence that axon terminals in visual cortex contain both acetylcholine and GABA (Beaulieu and Somogyi 1991) and further by the finding that boutons in the deep cerebellar nuclei contain GABA as well as glycine (Chen and Hillman 1993). Neuropeptides coexist with neurotransmitters (e.g. substance $P$ in GABAergic neurons, Jakab et al. 1997, see also review by Graybiel 1990). Co-release of these substances has been documented (Jonas et al. 1998). Based on observations after retrograde transport of ${ }^{3} \mathrm{H}$-aspartate combined with immunohistochemistry, White et al. (1994) proposed that striatal efferents may contain both glutamate and GABA. Boulland et al. (2004) were the first to surmise that in the hippocampus axon terminals exist that contain both
VGluT2-carrying synaptic vesicles and vesicles that carry vesicular GABA transporter. Most interesting is that dentate gyrus granule cells may be GABAergic in early stages of development and later switch to glutamate as their preferred neurotransmitter (Sloviter et al. 1996; review by Mody 2002). Thus, colocalization of an inhibitory and an excitatory neurotransmitter in one axon terminal may not be uncommon. Yet, completely unclear is what kind of neurophysiological events occur in GAD-VGluT2 axon terminals. Are GABA and glutamate co-released from these terminals simultaneously and from the same locus, or is release spatially and temporally separated? What is the proportion in one terminal between synaptic vesicles containing each individual neurotransmitter, what are possible other co-factors, and most fascinating, what are the effects on the postsynaptic neuron. All these questions provide as many challenges to the neuroscientist.

Acknowledgements The authors thank Nico Blijleven for technical assistance with the complicated Leica software, and Riichi Kajiwara for writing ImageJ scripts to perform threshold analysis. Part of this work was supported by the Interdisciplinary Center of Clinical Research (IZKF) at the Faculty of Medicine of the Universität Leipzig (project Z10).

\section{References}

Aihara Y, Mashima H, Onda H, Hisano S, Kasuya H, Hori T, Yamada S, Tomura H, Yamada Y, Inoue I, Kojima I, Takeda J (2000) Molecular cloning of a novel brain-type $\mathrm{Na}^{+}$-dependent inorganic phosphate co-transporter. J Neurochem 74:2622-2625

Alonso A (2002) Spotlight on the neurones (II): electrophysiology of the neurones in the peripheral and entorhinal cortices and neuromodulatory changes on firing patterns. In: Witter MP, Wouterlood FG (eds) The parahippocampal region, organisation and role in cognitive functions. Oxford University Press, Oxford, pp 89-105

Alonso-Nanclares L, Minelli A, Melone M, Edwards RH, DeFelipe J, Conti F (2004) Perisomatic glutamatergic axon terminals: A novel feature of cortical synaptology revealed by vesicular glutamate transporter 1 immunostaining. Neurosci 123:547-556

Arai R, Jacobowitz DM, Deura S (1994) Distribution of calretinin, calbindin-D28k, and parvalbumin in the rat thalamus. Brain Res Bull 33:595-614

Barroso-Chinea P, Castle M, Aymerich MS, Perez-Manso M, Erro E, Tunon T, Lanciego JL (2007) Expression of the mRNAs encoding for the vesicular glutamate transporters 1 and 2 in the rat thalamus. J Comp Neurol 501:703-15

Beaulieu C, Somogyi P (1991) Enrichment of cholinergic synaptic terminals on GABAergic neurons and coexistence of immunoreactive GABA and choline acetyltransferase in the same synaptic terminals in the striate cortex of the cat. J Comp Neurol 304:666-680

Beckstead RM (1978) Afferent connections of the entorhinal area in the rat as demonstrated by retrograde cell-labeling with horseradish peroxidase. Brain Res 152:249-264

Bellocchio EE, Hu H, Pohorille A, Chan J, Pickel VM, Edwards RH (1998) The localization of the brain-specific inorganic phosphate transporter suggests a specific presynaptic role in glutamatergic transmission. J Neurosci 18:8648-8659 
Bellocchio EE, Reimer RJ, Fremeau RT Jr, Edwards RH (2000) Uptake of glutamate into synaptic vesicles by an inorganic phosphate transporter. Science 289:957-60

Bertram EH, Zhang DX (1999) Thalamic excitation of hippocampal CA1 neurons: a comparison with the effects of CA3 stimulation. Neuroscience 92:15-26

Boulland JL, Qureshi T, Seal RP, Rafiki A, Gundersen V, Bergersen LH, Fremeau RT Jr, Edwards RH, Storm-Mathisen J, Chaudhry FA (2004) Expression of the vesicular glutamate transporters during development indicates the widespread corelease of multiple neurotransmitters. J Comp Neurol 480:264-80

Brecha N, Johnson D, Peichl L, Wässle H (1988) Cholinergic amacrine cells of the rabbit retina contain glutamate decarboxylase and $\gamma$-aminobutyrate immunoreactivity. Proc Natl Acad Sci USA 85:6187-6191

Burwell RD (2000) The parahippocampal region: corticocortical connectivity. Ann N Y Acad Sci 911:25-42

Burwell RD, Witter MP (2002) Basic anatomy of the parahippocampal region in monkeys and rats. In: Witter MP, Wouterlood FG (eds) The parahippocampal region, organisation and role in cognitive functions. Oxford University Press, Oxford, pp 35 -59

Buzsáki G (1996) The hippocampal-neocortical dialogue. Cerebr Cortex 6:81-92

Canteras NS, Simerly RB, Swanson LW (1994) Organization of projections from the ventromedial nucleus of the hypothalamus: a Phaseolus vulgaris-Leucoagglutinin study in the rat. J Comp Neurol 348:41-79

Chen S, Hillman DE (1993). Colocalization of neurotransmitters in the deep cerebellar nuclei. J Neurocytol 22:81-91

Dolleman-Van der Weel MJ, Lopes da Silva FH, Witter MP (1997) Nucleus reuniens thalami modulates activity in hippocampal field CA1 through excitatory and inhibitory mechanisms. J Neurosci 17:5640-50

Fremeau RT Jr, Troyer MD, Pahner I, Nygaard GO, Tran CH, Reimer RJ, Bellocchio EE, Fortin D, Storm-Mathisen J, Edwards RH (2001) The expression of vesicular glutamate transporters defines two classes of excitatory synapse. Neuron 31:247-60

Fremeau RT Jr, Voglmaier S, Seal RP, Edwards RH (2004) VGluTs define subsets of excitatory neurons and suggest novel roles for glutamate. Trends Neurosci 27:98-103

Freund TF, Martin KAC, Smith AD, Somogyi P (1983) Glutamate decarboxylase-immunoreactive terminals of Golgi-impregnated axoaxonic cells and of presumed basket cells in synaptic contact with pyramidal neurons of the cat's visual cortex. J Comp Neurol 221:263-278

Fujiyama F, Furuta T, Kaneko T (2001) Immunocytochemical localization of candidates for vesicular glutamate transporters in the rat cerebral cortex. J Comp Neurol 435:379-87

Gaykema RPA, Luiten PGM, Nyakas C, Traber J (1990) Cortical projection patterns of the medial septum-diagonal band complex. J Comp Neurol 293:103-124

Gloveli T, Schmitz D, Empson RM, Dugladze T, Heinemann U (1997) Morphological and electrophysiological characterization of layer III cells of the medial entorhinal cortex of the rat. Neuroscience 77:629-648

Gloveli T, Dugladze T, Schmitz D, Heinemann U (2001) Properties of entorhinal cortex deep layer neurons projecting to the rat dentate gyrus. Eur J Neurosci 13:413-20

Graybiel A (1990) Neurotransmitters and neuromodulators in the basal ganglia. Trends Neurosci 13:244-254

Härtig W, Riedel A, Grosche J, Edwards RH, Fremeau RT Jr, Harkany T, Brauer K, Arendt T (2003) Complementary distribution of vesicular glutamate transporters 1 and 2 in the nucleus accumbens of rat: Relationship to calretinin-containing extrinsic innervation and calbindin-immunoreactive neurons. J Comp Neurol 465:1-10
Hur EE, Zaborszky L (2005) VGluT2 afferents to the medial prefrontal and primary somatosensory cortices: a combined retrograde tracing in situ hybridization. J Comp Neurol 483:351373

Insausti R, Herrero MT, Witter MP (1997) Cytoarchitectonic subdivisions and the origin and distribution of cortical efferents. Hippocampus 7:146-83

Jakab RL, Goldman-Rakic P, Leranth C (1997) Dual role of substance P/GABA axons in cortical neurotransmission: synaptic triads on pyramidal cell spines and basket-like innervation of layer II-III calbindin interneurons in primate prefrontal cortex. Cereb Cortex 7:359-373

Jonas P, Bischofsberger J, Sandkuhler J (1998) Corelease of two fast neurotransmitters at a central synapse. Science 281:360 361

Jones RS, Buhl EH (1993) Basket-like interneurones in layer II of the entorhinal cortex exhibit a powerful NMDA-mediated synaptic excitation. Neurosci Lett 149:35-9

Kaneko T, Fujiyama F (2002) Complementary distribution of vesicular glutamate transporters in the central nervous system. Neurosci Res 42:243-250

Kemppainen S, Jolkkonen E, Pitkänen A (2002) Projections from the posterior cortical nucleus of the amygdala to the hippocampal formation and parahippocampal region in rat. Hippocampus $12: 735-55$

Kloosterman F, van Haeften T, Lopes da Silva FH (2004) Two reentrant pathways in the hippocampal-entorhinal system. Hippocampus 14:1026-1039

Kumar SS, Buckmaster PS (2006) Hyperexcitability, interneurons, and loss of GABAergic synapses in entorhinal cortex in a model of temporal lobe epilepsy. J Neurosci 26:4613-1423

Lorente de Nó R (1933) Studies on the structure of the cerebral cortex. I. The area entorhinalis. J Psychol Neurol (Leipzig) 45:381-438

Miettinen M, Koivisto E, Riekkinen P, Miettinen R (1996). Coexistence of parvalbumin and GABA in nonpyramidal neurons of the rat entorhinal cortex. Brain Res 706:113-22

Miettinen M, Pitkänen A, Miettinen R (1997) Distribution of calretinin-immunoreactivity in the rat entorhinal cortex: coexistence with GABA. J Comp Neurol 378:363-378

Mody I (2002) The GAD-given right of dentate gyrus granule cells to become GABAergic. Epilepsy Curr 2:143-145

Ni B, Rosteck PR Jr, Nadi NS, Paul SM (1994). Cloning and expression of a cDNA encoding a brain-specific $\mathrm{Na}^{+}$dependent inorganic phosphate cotransporter. Proc Natl Acad Sci USA 91:5607-5611

Oertel WH, Schmechel DE, Tappaz ML, Kopin IJ (1981) Production of a specific antiserum to rat brain glutamic acid decarboxylase by injection of an antigen-antibody complex. Neuroscience 6:2689-700

Oertel WH, Mugnaini E, Schmechel DE, Tappaz ML, Kopin IJ (1982) The immunocytochemical demonstration of gamma aminobutyric acidergic neurons. Method and application. In: Chan-Palay V, Palay SL (eds) Cytochemical methods in Neuroanatomy. Alan R. Liss, New York, pp 297-329

Ottersen OP, Storm-Mathisen J (1984) Glutamate- and GABAcontaining neurons in the mouse and rat brain, as demonstrated with a new immunocytochemical technique. J Comp Neurol 229:374-392

Pitkänen A (2000) Connectivity of the rat amygdaloid complex. In: Aggleton JP (ed) The amygdala: a functional analysis, 2nd edn. Oxford University Press, Oxford, pp 31-115

Rasband WS (1997-2006) ImageJ, U. S. National Institutes of Health, Bethesda, Maryland, USA, http://rsb.info.nih.gov/ij/

Résibois A, Rogers JH (1992) Calretinin in rat brain: an immunohistochemical study. Neuroscience 46:101-134 
Sloviter RS, Dichter MA, Rachinsky TL, Dean E, Goodman JH, Sollas AL, Martin DL (1996) Basal expression and induction of glutamate decarboxylase and GABA in excitatory granule cells of the rat and monkey hippocampal dentate gyrus. J Comp Neurol 373:593-618

Steward O, Scoville SA (1976) Cells of origin of entorhinal cortical afferents to the hippocampus and fascia dentata of the rat. J Comp Neurol 169:347-370

Swanson LW, Köhler C (1986) Anatomical evidence for direct projections from the entorhinal area to the entire cortical mantle in the rat. J Neurosci 6:3010-23

Takamori S, Rhee JS, Rosenmund C, Jahn R (2001) Identification of differentiation associated brain-specific phosphate transporter as a second vesicular glutamate transporter (VGluT2). J Neurosci 21: RC 182(1-6)

van der Voort HTM, Strasters KC (1995). Restoration of confocal images for quantitative image analysis. J Microsc 158:43-45

van Groen T, Wyss JM (1995) Projections from the anterodorsal and anteroventral nucleus of the thalamus to the limbic cortex in the rat. J Comp Neurol 358:584-604

van Haeften T, Baks-te-Bulte L, Goede P, Wouterlood FG, Witter MP (2003) Morphological and numerical analysis of synaptic interactions between neurons in the deep and superficial layers of the entorhinal cortex of the rat. Hippocampus 13:943-952

Vertes RP, Hoover WB, Do Valle AC, Sherman A, Rodriguez JJ (2006) Efferent projections of reuniens and rhomboid nuclei of the thalamus in the rat. J Comp Neurol 499:768-96

White LE, Hodges HD, Carnes KM, Price JL, Dubinsky JM (1994) Colocalization of excitatory and inhibitory neurotransmitter markers in striatal projection neurons in the rat. J Comp Neurol 339:328-340

Witter MP (2002) The parahippocampal region: past, present and future. In: Witter MP, Wouterlood FG (eds) The parahippocampal region, organisation and role in cognitive functions. Oxford University Press, Oxford, pp 3-19

Witter MP, Amaral DG (2004) The hippocampal region in the rat brain. In: Paxinos $\mathrm{G}$ (ed) The rat brain, 3rd edn. Elsevier, San Diego, pp 637-703

Wouterlood FG (2002) Spotlight on the neurons: cell types, local connectivity, microcircuits and distribution of markers. In: Witter MP, Wouterlood FG (eds) The parahippocampal region, organisation and role in cognitive functions. Oxford University Press, Oxford, pp 61-88
Wouterlood FG (2006) Combined fluorescence methods to determine synapses in the light microscope: multilabel confocal laser scanning microscopy. In: Zaborszky L, Wouterlood FG, Lanciego JL (eds) Neuroanatomical tract-tracing 3: Moleculesneurons-systems. Springer, New York, pp 394-435

Wouterlood FG, Mugnaini E, Nederlof J (1985) Projection of olfactory bulb efferents to layer I GABA-ergic neurons in the entorhinal area. Combination of anterograde degeneration and immunoelectron microscopy in rat. Brain Res 343:283-296

Wouterlood FG, Saldana E, Witter MP (1990) Thalamic afferents to the hippocampal region studied with the anterograde tracer Phaseolus vulgaris-leucoagglutinin. Light and electron microscopy. J Comp Neurol 296:179-203

Wouterlood FG, Härtig W (1995) Calretinin-immunoreactivity in mitral cells of the rat olfactory bulb. Brain Res 682:93-100

Wouterlood FG, Härtig W, Brückner G, Witter MP (1995) Parvalbumin-immunoreactive neurons in the entorhinal cortex of the rat: localization, morphology, connectivity and ultrastructure. J Neurocytol 24:135-153

Wouterlood FG, van Denderen J, van Haeften T, Witter MP (2000) Calretinin in the entorhinal cortex of the rat: distribution, morphology, ultrastructure of neurons, and co-localization with $(\gamma$-amino butyric acid and parvalbumin. J Comp Neurol 425:177-192

Wouterlood FG, van Haeften T, Blijleven N. Perez-Templado P, Perez-Templado E (2002) Double-label confocal laser scanning microscopy, image restoration and real-time 3D-reconstruction to study axons in the CNS and their contacts with target neurons. Appl Immunohistochem Mol Morphol 10:85-102

Wouterlood FG, Canto C, Grosche J, Witter MP, Härtig W (2003) Vesicular glutamate transporter 2 expressed in a subpopulation of calretinin-containing axons in rat entorhinal cortex. Abstract nr. 155.9, 2003 Abstract Viewer/Itinerary Planner. Society for Neuroscience, Washington, DC (online)

Wouterlood FG, Boekel AJ, Meijer GA, Beliën JAM (2007a) Computer assisted estimation in the CNS of 3D multimarker "overlap" or "touch" at the level of individual nerve endings. A confocal laser scanning microscope application. J Neurosci Res 85:1215-1228

Wouterlood FG, Aliane V, Boekel AJ, Hur E, Zaborszki L, Pedro Barroso-Chinea P, Härtig W, Lanciego JL, Witter MP (2007b) Origin of calretinin containing, vesicular glutamate transporter 2-coexpressing fiber terminals in the entorhinal cortex of the rat. J Comp Neurol (in press) 